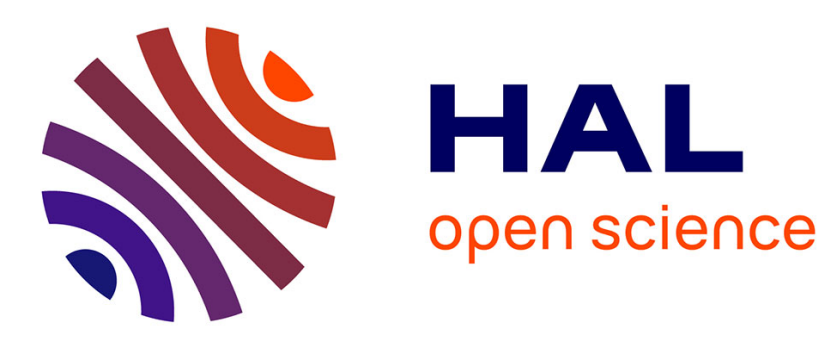

\title{
WoaH: A Virtual Reality Work-at-Height Simulator
}

Cédric Di Loreto, Jean-Rémy Chardonnet, Julien Ryard, Alain Rousseau

\section{To cite this version:}

Cédric Di Loreto, Jean-Rémy Chardonnet, Julien Ryard, Alain Rousseau. WoaH: A Virtual Reality Work-at-Height Simulator. 2018 IEEE Conference on Virtual Reality and 3D User Interfaces, Mar 2018, Reutlingen, Germany. pp.281-288, 10.1109/VR.2018.8448292 . hal-01933284

\section{HAL Id: hal-01933284 https://hal.science/hal-01933284}

Submitted on 17 Mar 2021

HAL is a multi-disciplinary open access archive for the deposit and dissemination of scientific research documents, whether they are published or not. The documents may come from teaching and research institutions in France or abroad, or from public or private research centers.
L'archive ouverte pluridisciplinaire HAL, est destinée au dépôt et à la diffusion de documents scientifiques de niveau recherche, publiés ou non, émanant des établissements d'enseignement et de recherche français ou étrangers, des laboratoires publics ou privés. 


\section{WoaH: A Virtual Reality Work-at-Height Simulator}

\author{
Cédric Di Loreto* \\ LISPEN, Arts et Métiers, \\ UBFC, HESAM, Institut \\ Image, France \\ Enedis Bourgogne, France
}

\author{
Jean-Rémy Chardonnet ${ }^{\dagger}$ \\ LISPEN, Arts et Métiers, \\ UBFC, HESAM, Institut \\ Image, France
}

\author{
Julien Ryard \\ LISPEN, Arts et Métiers, \\ UBFC, HESAM, Institut \\ Image, France
}

\author{
Alain Rousseau ${ }^{\S}$ \\ Enedis Bourgogne, France
}

\begin{abstract}
We present WoaH, a virtual reality work-at-height simulator aimed at (i) testing whether future workers are able to manage their stress when high up and thus easily detect susceptibility to vertigo, and (ii) training in a typical work-at-height engineering operation. The simulator is composed of a real ladder synchronized in position with a virtual one placed 11 meters above the ground in a virtual environment. Visualization is done through a head-mounted display (HMD). We conducted a first user study evaluating our simulator in terms of cybersickness, perceived realism and anxiety, through both subjective (questionnaires) and objective (electrodermal activity) measurements, and testing whether vibratory cues could enhance the level of anxiety felt. Results indicate that WoaH generates anxiety as expected and is perceived as realistic. Adding vibrations had significant impact on the perceived realism but not on the electro-dermal activity. These first results bring insights to future developments for a deployment in companies dealing with work at height.
\end{abstract}

Index Terms: Human-centered computing-Human computer interaction (HCI) - Interaction paradigms - Virtual reality; Software and its engineering - Software organization and properties-Virtual worlds software-Virtual worlds training simulations

\section{INTRODUCTION}

The use of virtual reality (VR), especially by companies, has become more and more common in the past few years, thanks to the availability of low cost devices, such as the Oculus Rift or the HTC Vive head-mounted displays (HMD). Virtual reality enables to immerse a user into a virtual environment and allows to evolve in a reproducible and safe way in situations that could be dangerous in reality. It can therefore be used as a tool to train, control, prevent or even treat disorders. For instance, on one hand, virtual reality exposure therapy was proposed a few decades ago to treat fear of heights. On the other hand, the entertainment field took the opportunity of low cost HMDs to propose experiencing fear of heights and vertigo in various situations such as walking on a board suspended high above the ground, or virtual rock climbing. From a more real applicative point of view, work-at-height is one of the dangerous situations encountered by workers that can be reproduced using virtual reality [16]. Companies can make use of such technology as a prevention tool to check the ability of their workers to work at height or to train them with full immersion in realistic conditions, without being exposed to possible fatal issues while reducing insurance costs and space needed compared to real work-at-height simulation mockups. However, still very few VR-based simulators are available for such use cases [16].

We present WoaH, a transportable virtual reality Work-at-Height simulator (see Fig. 1), aimed at testing whether users are able to

\footnotetext{
*e-mail: cedric.di-loreto@ensam.eu

${ }^{\dagger}$ Member, IEEE, e-mail: jean-remy.chardonnet@ensam.eu

†e-mail: julien.ryard@ensam.eu

§e-mail: alain-r.rousseau@enedis.fr
}

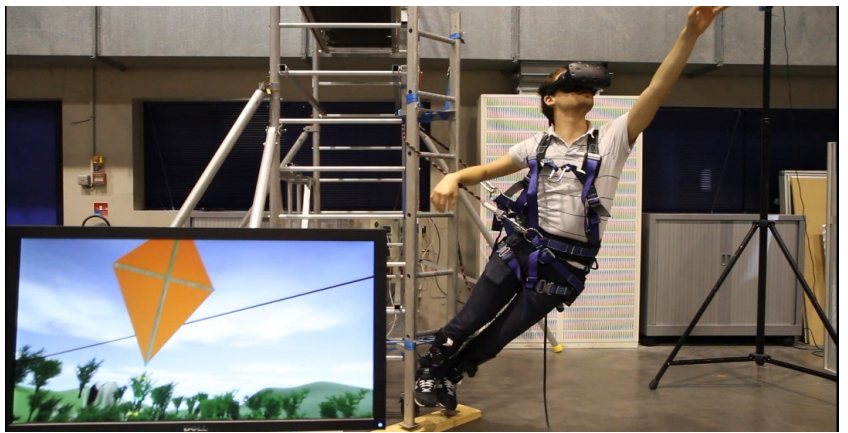

Figure 1: The WoaH simulator in operation.

manage their stress during the simulation of a typical work-at-height operation or not. Our motivation lies in investigating the parameters influencing the fear of heights in a virtual environment and allowing an accurate restitution of a real work-at-height situation. Therefore, in this study, we mainly focused on presence, more specifically the perceived realism, and anxiety, as they may represent strong indicators to judge the simulator's performance. For that purpose, multisensory cues were integrated. The final goal of WoaH is to be deployed in companies dealing with work at height, as an additional tool to hire workers, limitating risks and cost. Here, the use case of the WoaH simulator concerns the maintenance of high-voltage power lines.

\subsection{Related Work}

\subsubsection{Vertigo and Psychology}

Vertigo has been widely studied in the last decades. Two different kinds of vertigo can be distinguished [4,7]: the distance vertigo, seen as physiological, and acrophobia, seen as psychological and thus basically as a fear. Brandt et al. focus on the physiological height vertigo and show that the eye-object distance is the main parameter affecting vertigo. A conflict between visual and vestibular information was hypothesized to be responsible for vertigo. Humans continuously need visual cues to stabilize themselves. The parallax induced by natural displacements of the head helps stabilizing. When a subject is high up, the lack of close and stable visual references involves a parallax that is under the eye perception threshold. Therefore it imposes him to rely on his proprioceptive and vestibular information to stabilize himself, resulting in possible increased body sway. Coelho and Wallis further support this theory by asserting that a larger sway may generate a fear of falling and so the sensation of vertigo [11]. Acrophobic subjects may be persons with vestibular system deficiency or for whom visual information are too important. Therefore stabilization of their body becomes difficult and implies a larger body sway. Experimental studies revealed that vertigo may be a means for the body to indicate a situation inducing postural instability and so possibly leading to a fall. Moreover, Brandt et al. propose a trigonometrical model for distance vertigo [7]. Based on this model, the distance of five meters is announced as a saturation distance for visual cues from which body sway does not increase 
anymore [11]. The effect of body postures has also been studied and results show that standing up in front of the void is the posture increasing the sensation of vertigo at most.

Coelho et al. highlighted the impact of another parameter on the variation of anxiety in height-involving situations, that is displacements [10]. Results show that especially lateral displacements induce more anxiety on acrophobic subjects. Adding lateral displacements in a virtual simulation may therefore increase anxiety on acrophobic subjects, but also the simulation susceptibility to trigger the sensation of vertigo, along with significant variations of physiological and behavioral reactions from subjects.

Cohen proposed a subjective method to evaluate the inclination to acrophobia, through a dedicated questionnaire [13]. Results show that a relationship does exist between the reported acrophobia score and the level of anxiety.

\subsubsection{Acrophobia in Virtual Reality}

Since virtual reality exists, several studies have been conducted on height perception in virtual environments and explored possibilities to use it to treat acrophobia.

Cleworth et al. conducted an experiment reproducing exactly the same anxiogenic situation in the real world and in virtual reality [9]. Measuring electrodermal activity, anxiety, fear and movements of the center of pressure of participants, it appears that these measures significantly increase with height, but their variations are less important in a virtual exposure compared to an in vivo exposure.

Using a CAVE system, Simeonov et al. showed that virtual reality appears as a good tool to reproduce the psychological and physiological effects of height-involving situations on subjects [35]. Typical applications include prevention or control in situations where people work at height.

Other past work showed that HMDs can also reproduce the symptoms of a height exposure. Hüweler et al. submitted healthy and acrophobic subjects to a visual stimulus aiming at inducing a conflict between visual and somatosensory information [17]. Highly acrophobic subjects reported more anxiety and vertigo than healthy subjects, showing that receiving contradictory information is enough to induce anxiety on acrophobic subjects, thus supporting vertigo mechanisms theories previously exposed in $[4,7]$.

As past work showed the efficiency of virtual reality in reproducing physiological symptoms of a real exposure to height, researchers explored possibilities to use virtual reality for the treatment of acrophobia. The first studies date back to 1995 and showed promising results [34]. In this regard, virtual reality has shown to be at least as effective as an in vivo exposure for the treatment of acrophobia $[10,15,18]$. Based on previous findings, Coelho et al. emphasize the role of lateral displacements during an acrophobia therapy, involving the role of parallax in the visuo-vestibular conflict which occurs in the distance vertigo [12]. In this paper, we do not aim at providing a simulator for treating acrophobia, but just to use it as a detection tool of acrophobia and a training tool for real dangerous situations.

\subsubsection{Presence and Anxiety}

Presence is another parameter to take into account to ensure a simulator's fidelity. Slater and Wilbur define the presence as the sense of being here [39]. Presence should make subjects act as if they were in a real environment. They should remember the experiment more as a place they visited than as pictures they saw. Slater further investigated this aspect and proposed two concepts contributing to realistic behaviors in immersive virtual environments: Place Illusion and Plausability Illusion [37], further developed in [36].

Juan and Pérez compared the level of presence and anxiety in a height-involving situation with an HMD and a CAVE system [19]. Though with both systems a positive correlation between anxiety and presence was revealed, better results have been significantly obtained with the CAVE system. However, the HMD used in this work had an only $40^{\circ}$ field of view, which is much less than what current HMDs propose. In our case, we aim at providing a transportable simulator. Therefore, large-screen displays have not been considered for our simulator (compared to the one presented in [16]), as they are hardly transportable. Moreover, in work-at-height conditions, subjects may need to look up. When using a CAVE system or another large-screen display, it requires the display system to have at least a ceiling or high screens, which is not necessary the case of most existing systems. This issue does not arise when using an HMD.

Some studies found contradictory results on the relation between presence and anxiety [1,6, 22, 26, 32]. Early studies showed anxiety not to significantly correlate with presence [32], however the hardware used consisted in a monoscopic color HMD which is not appropriate. Krijn et al. do not find a relation between anxiety and presence [22]. They rather suggest that a minimum of presence is required for subjects to develop anxiety but more presence does not necessarily generate more anxiety. However, Bouchard et al. found that anxiety increases the sense of presence in stressful environments [6], though the phobia considered in their study was not related to a fear of heights. Alsina-Jurnet et al. suggest that presence and anxiety are not linked in non-stressful environments but this relation does exist in stressful environments, especially for acrophobic people [1]. In stressful environments, the relationship between presence and anxiety would be, the more presence, the more anxiety, which was also found in previous studies (for example [26] where anxiety could be used as a proxy measure for presence). This study also reveals that the main parameter for anxiety to develop is the perceived realism of the virtual environment (which seems to be a factor corresponding more to Slater's Plausability Illusion). This factor has been well studied in past research related to social interactions (see for example [23]) showing that visual quality significantly influences anxiety.

\subsubsection{Vertical Navigation and Impact of Multisensory Cues}

In work-at-height situations, subjects may need to move up and down in the virtual environment, which has been hardly addressed in past work, as one of the issues is for instance to render physical efforts engendered by gravity while climbing. Slater et al. were one of the first to propose navigation methods based on body centered interaction to climb stairs and ladders, aiming at reducing the mismatch between visual and proprioceptive information [38]. Lai et al. used the march and reach technique to virtually climb a ladder [24]. For WoaH, we want users to feel real sensations of height and physical efforts. In the use case we address, only a few steps - two or three steps maximum - need to be climbed. Therefore, rather than tricking users with artificial travel techniques, we propose climbing a real ladder to strengthen immersion.

Multisensory (visual, haptic) cues may influence the simulator's performance on immersion and presence. In this regard, a huge piece of work in the literature has been provided for applications not implying vertical motion (see for example [14]). As we aim at providing a transportable simulator, light devices able to provide multisensory cues should be considered. For instance, Plouzeau et al. showed the efficiency of vibrations in classical navigation [30]. It also highlighted the contribution of vibrations on the sense of presence during navigation in virtual environments. Vasyleska and Kaufmann studied the effect of three different metaphors for vertical navigation [41]. The best results in terms of presence were obtained by using a vibrant platform. They reproduced the atmosphere of an elevator which is used as a metaphor for subjects to navigate between floors.

\subsection{Contributions}

We present here first results of our work-at-height simulator. WoaH aims at being used by companies to detect acrophobia on potential 
future workers and to train them in real work-at-height operations. To the best of our knowledge, we are not aware of such VR-based simulators, apart from the one presented in [16] which has however different caracteristics and addresses a different work-at-height situation as we do. By providing the WoaH simulator, we try to fill this gap and match as much as possible real work-at-height situations. For that purpose, we investigate the parameters influencing the fear of heights in a virtual environment and allowing high fidelity in the simulation. We propose to use a real ladder allowing a perfect match in terms of body sensations and physical efforts between the real and the virtual environments, as past research showed an enhancement in the simulation when including real objects [16]. Compared to past work related to vertical navigation in virtual environments, we chose not to trick users as we believe that producing physical efforts will enhance the level of immersion, the perceived realism, and trigger anxiety much easier. We propose also to test multisensory modalities that can potentially increase the subjects' sense of presence and the likelihood of provoking anxiety among them. Here we integrated vibrations and the ability to see the hands and feet of the subjects' virtual avatar. The motivation behind studying the effect of vibrations are the past results showing the increase of the sense of presence. Moreover, it is expected that, as vertigo is closely linked to postural stability, such a modality could also induce an increase in the anxiety felt by the subjects. Visual feedback was added as a visual guide to facilitate the tasks to achieve (especially climbing on a ladder), as subjects are totally immersed in the virtual environment leading them to use proprioception.

\section{THE WOAH SIMULATOR}

\subsection{Hardware}

We aim at providing a transportable simulator. The reasons for such feature lie in a request from a company dealing with the maintenance of electrical power lines we worked with, to be easily installed and uninstalled in various locations of the company throughout the country, enabling efficient on-site training. Pros of a transportable simulator compared to a stationary one include its light weight yet still enabling full immersion, cost effectiveness, ease of use and easy duplicability. Cons are the need to calibrate the simulator each time it is installed, and less visual quality in terms of resolution than with large systems with high resolution capabilities.

To be transportable, we opted for an HTC Vive HMD as a visualization device, coupled with a VR-Ready Windows 10 PC. Though HMDs shield users from reality, it allows to be totally immersed in realistic situations, which would not have been possible with a real ladder simulator or using see-through displays as users would have been influenced by the surrounding environment. An audio headset supplements the system for phonic immersion. Four Vive trackers are placed on the tibia and the hands of the user with a simple home-made support, since they represent a very good compromise between cost, robustness and low latency $(10 \mathrm{~ms})$, avoiding its effect on presence, especially in stressful environments [27]. The trackers are mainly used to provide visual feedback of the hands and feet positions to users.

A home-made 3-degree-of-freedom dynamic platform [29] was integrated to enhance the performance of the simulator in terms of immersion, by reproducing the vibrations of a virtual aerial device during its motion. This dynamic platform is composed of four electric actuators ( $8 \mathrm{~cm}$ of maximal displacement) and each of them can be controlled independently from the others. The platform is able to perform motion along the vertical axis and around the pitch and roll axes. The actuators can also reproduce vibrations at frequencies up to $3 \mathrm{kHz}$.

A real ladder is attached to a pole so that users can climb it for real during the operation. The real ladder is synchronized in position with its virtual representation in the virtual environment during the simulator's installation. Users are equipped with a harness for their

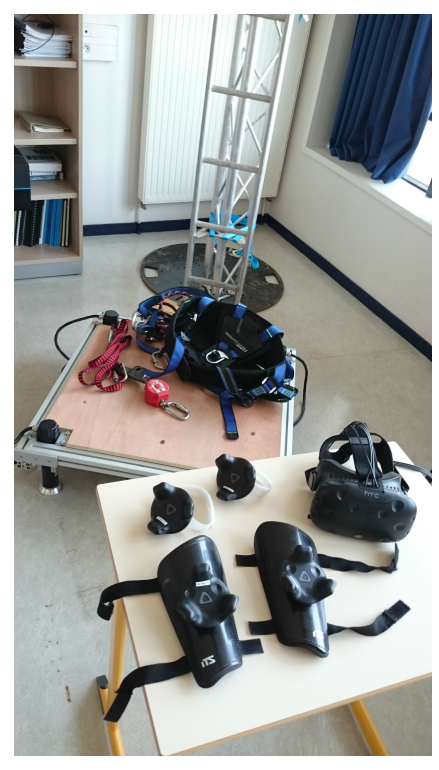

Figure 2: The WoaH simulator setup. In the foreground, the HTC Vive with the trackers to be attached to the user; in the background, the dynamic platform with the harness to be attached to the user and the real ladder fixed on a lightweight structure.

safety that allows them to evolve hands-free on the ladder. We chose to put a real ladder to get a perfect matching between the real and the virtual environments in terms of body sensations (e.g., sensation of gravity) and physical efforts. This also explains the reason to choose an HMD as a visualization device rather than a CAVE system for instance, as the total setup takes some space in terms of height.

The whole hardware setup is depicted in Fig. 2. As mentioned, WoaH has been designed to be transportable. Therefore, the ladder can be easily fixed on and removed from the pole while the pole is part of a lightweight structure that is easily mountable and demountable. The whole simulator takes about 45 minutes to be set up and calibrated.

\subsection{The Virtual Environment Design}

The WoaH simulator uses a specific scene developed under Unity (see Fig. 3). In this scene, subjects are placed on the platform of a virtual aerial device. The dynamic platform and the platform of the aerial device match in position, therefore subjects can feel for real the void with their feet on the edge. During the rise of the aerial device, vibrations can be set on the dynamic platform. Here, they have been set to an amplitude of $1 \mathrm{~cm}$ and at a frequency of $1200 \mathrm{~Hz}$. These parameters were chosen empirically from several trials with a real aerial device. The motion of the aerial device's platform integrates also a lateral component, which is prone to anxiety according to past work [10] and may affect the perception of height [12]. As soon as the aerial device's platform is in its upper position, the dynamic platform jerks once in a short time to reproduce the stop of the aerial device, and then starts swaying in every direction (amplitude: $2 \mathrm{~cm}$, frequency: $0.3 \mathrm{~Hz}$ ). This sway has been implemented to reproduce the effect of the wind on the aerial device, generating more stress to subjects as they may feel less stable. The aerial device's motion is set so that once elevated, subjects can step physically onto a virtual ladder placed on an electric pole. As mentioned earlier, the virtual ladder is synchronized in position with the real ladder so that subjects can climb the real ladder even though they do not see it because of the HMD. The virtual aerial device rises to an altitude of 11.6 meters. The surrounding environment is above 10 meters in distance in order not to give any static spatial references that could 


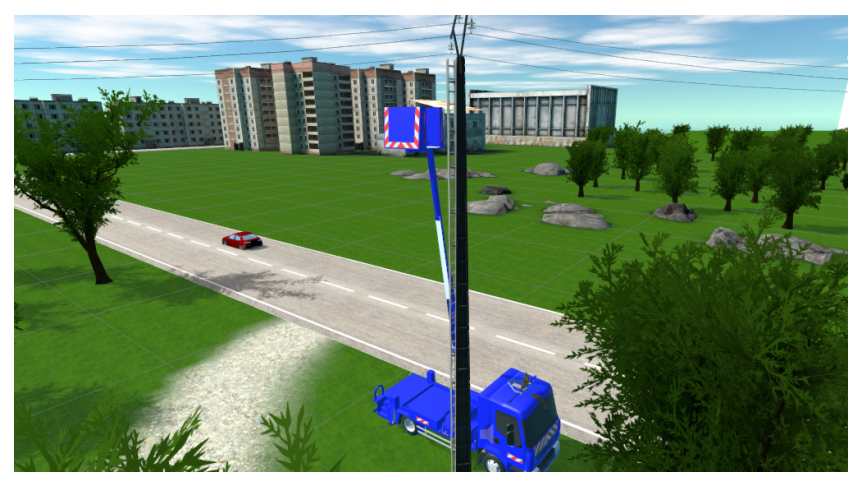

Figure 3: Global view of the scene.

be used to stabilize $[4,7]$.

Moving clouds have been added to the sky to strengthen the effect of loss of static spatial references. The surrounding environment is composed of buildings, rocks, hills and trees that are far enough from the subjects, again not to provide any static spatial references. Dynamism is included in the scene thanks to moving cars and 3D sounds emitted by cars, birds and the aerial device.

Finally, because subjects are cut from reality due to the use of an HMD, we included the possibility for subjects to see a virtual representation of their hands and feet as a visual guide. We chose as a first implementation to represent only hands and feet to simplify motion tracking computation. These parts were just tracked in position and not kinematically animated.

\section{USER STUDY}

A within-subject study was designed to assess the performance of the simulator. The tasks to achieve have been planned in cooperation with the company we worked with and were based on typical operations performed by their employees. We hypothesized the following:

H1 Multisensory cues increase the level of immersion and the sense of presence

H2 Multisensory cues increase the level of anxiety felt

\subsection{Participants}

14 participants (13 males and 1 female, ages: $21-27$ (mean: 23.4)) took part to the study. Only one of them had never experienced a VR system before the experiment. They were recruited within the university members and through word of mouth. No compensation was given at the end of the experiment. A briefing was provided individually to give enough information about the test procedure and possible risks before each experiment. Note that in this study, we voluntarily chose to recruit participants who were not familiar with work-at-height situations to test our simulator's performance in terms of immersion and anxiety.

All participants were asked to fill a demographic form with personal information and questions on particular health issues. None of them reported having particular health issues and were in their usual state of fitness.

They were asked to answer the Acrophobia Questionnaire from Cohen [13] to detect participants with possible acrophobia. From this questionnaire, 11 participants got an acrophobia score under 15 , which is low considering that the maximum score for this questionnaire is 120 .

\subsection{Experimental Protocol}

Two modalities were tested: without any vibration from the dynamic platform (M1) and with vibrations (M2). Participants did both

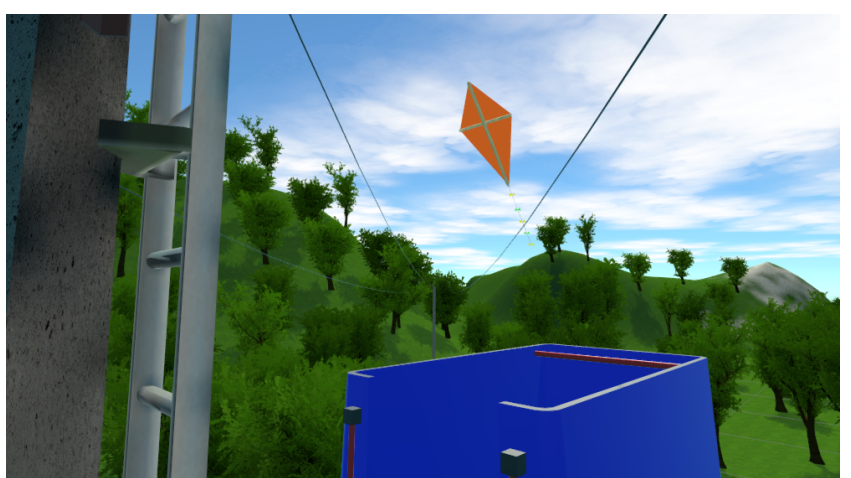

Figure 4: Position of the kite to be caught.

sessions, one per day with a different modality each time to prevent cumulative aspects. The modalities were distributed among the participants to avoid any bias of learning effects on the results. Each session lasted around 15 minutes on average. The protocol was as follows:

1. participants are helped to put on all the equipment (harness, trackers, head-mounted display, audio headset);

2. they are then placed on the dynamic platform and a one-minute familiarization session begins to get used to the trackers and virtual reality. After familiarization ends, a fade-in/fade-out effect allows a smooth transition to the main scene;

3. participants are placed on the platform of the virtual aerial device. They are able to watch the environment of the scene for 20 seconds before the rise of the aerial device starts. The rise of the aerial device is triggered by the experimenter through a user interface displayed on a separate screen;

4. once the aerial device's platform reaches its final position, participants are asked to step physically onto the ladder and climb one step;

5. the experimenter comes to harness the participants to the ladder and the pole. Though it may not look realistic, in real training, trainees may be assisted by an experienced person. Once harnessed, participants are able to move hands-free safely;

6. the aerial device moves off the ladder so that participants cannot step again onto the platform of the aerial device;

7. participants are asked to try to catch a kite placed behind them using both hands (see Fig. 4). Though typical operations on electrical poles are not to catch kites, this task has been included to check whether participants are confident in the harness, which is a requisite for workers to work at height. Indeed, to catch the kite, participants need to turn around, completely removing both hands off the ladder (see Fig. 1). Also, we voluntarily chose a simple task, following the company's recommendation as by experience, strongly acrophobic people would stop the session even before performing this task;

8. the experiment ends as soon as the kite is caught.

At anytime, participants were able to stop the experiment if necessary (e.g., sickness felt, vertigo). 

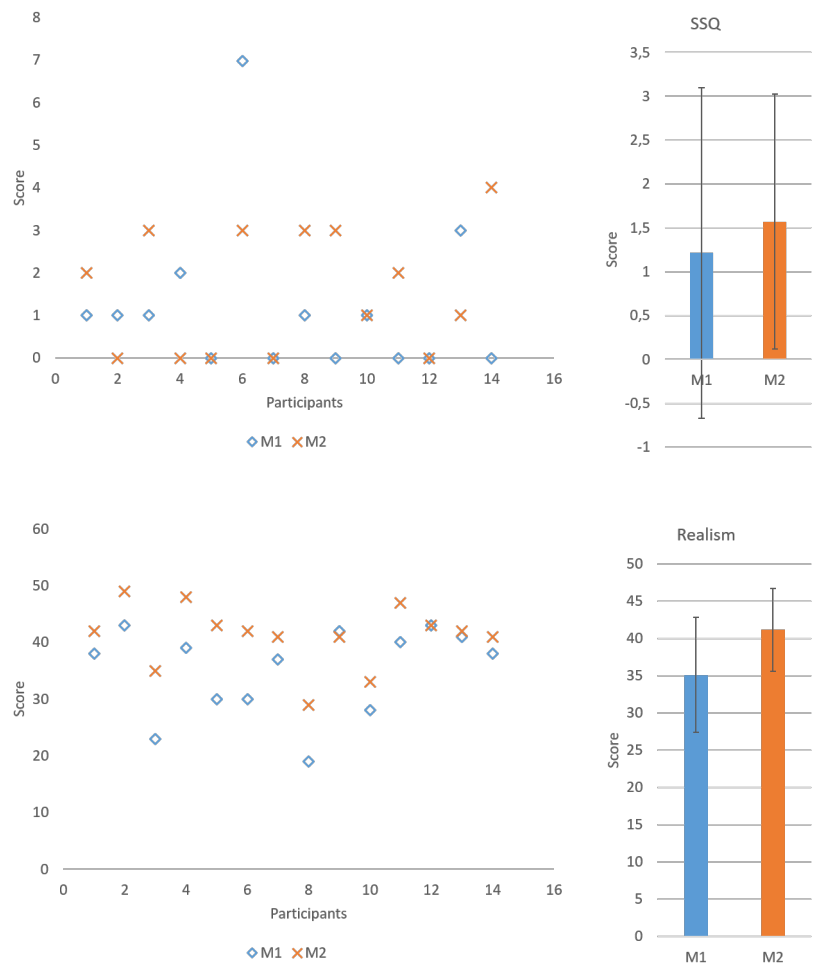

Figure 5: For each modality (M1: without any vibrations; M2: with vibrations), Top: SSQ scores; Bottom: Realism scores.

\subsection{Measurements}

Throughout the experiment, participants' electrodermal activity (EDA) was measured using an E4 Empatica wristband that can be remotely controlled by Wifi, with a sampling rate of $4 \mathrm{~Hz}$ and a precision about $0.01 \mu \mathrm{S}$ [25]. Other physiological measurements, such as blood pressure, heart rate, or behavioral measurements, such as postural stability, could have been considered. However, these require more intrusive devices than EDA wristbands, though in a fear of heights scenario, heart rate has shown to perform better than skin conductance [26]. Moreover, for example, the analysis of postural sway may be difficult to perform as: (i) this measure can be an indicator of combined factors such as cybersickness [8], anxiety [31], (ii) we created a situation where steady visual references may be partially lost, thus implying potential destabilization of participants' body [7], and so potentially biasing the measures. The EDA wristband records the participants' sweating, which gives an objective indication of the anxiety felt by the participants [3]. The wristband records data throughout the experiment.

At the end of the experiment, participants were asked to fill out two questionnaires: Kennedy et al.'s Simulator Sickness Questionnaire (SSQ) [20] to measure potential cybersickness induced by vertical navigation, and a perceived realism questionnaire derived from Witmer-Singer's presence questionnaire [42] to measure the level of the perceived realism of the scenario (we used only the questions related to the realism factor from the presence questionnaire). Oral comments were also recorded to get participants' feelings regarding anxiety.

At the end of the last session, participants were asked to rank the modalities according to their preference in terms of realism, level of immersion, and to freely comment their ranking.

\section{Results \\ 4.1 Cybersickness}

From the SSQ questionnaires, sickness scores were calculated using the method provided by Kennedy et al. [20]. A Mann-Whitney test was conducted to compare the effect of vibratory cues. No significant effect was found on cybersickness $(p=.359)$ between the modality with vibrations $(M=1.57, S D=1.45)$ and the one without any vibrations $(M=1.21, S D=1.89)$ (see Fig. 5 top).

SSQ scores are very low. In fact, many participants reported on the SSQs no sickness at all after the sessions. Moreover, observations made during the sessions showed that none of the participants complained about sickness or looked sick or confused after the experiment. This shows our simulator does not generate any strong sickness, which is an important feature for the simulator to be deployed.

\subsection{Perceived Realism}

We used only the realism factor questions from the presence questionnaire, as from the literature, the main parameter for anxiety to develop is the perceived realism of the virtual environment [1], and the perceived realism was found to have a significant impact on presence [5]. A Mann-Whitney test was conducted to compare the effect of vibrations on the perceived realism. There was a significant effect of vibrations on the perceived realism $(p=.023)$. The perceived realism was significantly higher with vibrations $(M=41.1, S D=5.6)$ than without any vibrations $(M=35.1, S D=7.7)$ (see Fig. 5 bottom).

According to the rankings that were asked to the participants at the end of the sessions, most of the participants preferred the modality including vibrations in terms of immersion (13 participants out of 14). The presence of a visual feedback on the hands and feet was appreciated by the participants as they could rely not only on their proprioception to move in the virtual environment, thus facilitating them in achieving the required tasks.

\subsection{Electrodermal Activity}

Recall that we measured the electrodermal activity to evaluate the level of anxiety. As the data did not follow a normal distribution, they were transformed using a Box-Cox transformation. A Student's t-test was conducted to compare the effect of vibrations on the EDA. No significant effect was found on the EDA $(p=.302)$ between the modality with vibrations $(M=7.7 \mu S, S D=11.7)$ and the one without any vibrations $(M=2.6 \mu S, S D=2.7)$ (see Fig. 6A).

Five participants orally indicated that the vertigo they felt during the sessions was higher when with vibrations. During the experiment, tremors have been observed on three participants while on the ladder, indicating not only a fear of height but also strong immersion in the virtual environment. For these participants, high EDA variations along with a high increase in the EDA have been observed, showing that our simulator indeed induced anxiety. Figures $6 \mathrm{~B}$ and $\mathrm{C}$ show the evolution of the EDA for one non-acrophobic participant and for the four participants who reported being acrophobic from the Acrophobia Questionnaire. We clearly see that for the non-acrophobic participant, the EDA remains low with very low variations throughout the experiment. Whereas, for the acrophobic participants, the EDA increases much more with high variations, especially when climbing to the ladder, turning around and removing both hands off the ladder to catch the kite.

As past literature showed that the EDA could be an indicator of cybersickness [21], we checked whether here the level of the EDA could be correlated to the SSQ scores. No correlation was found between the increase in the EDA and the SSQ scores $(r=-.03)$, showing that the increase in the EDA is rather due to anxiety and not to cybersickness. 

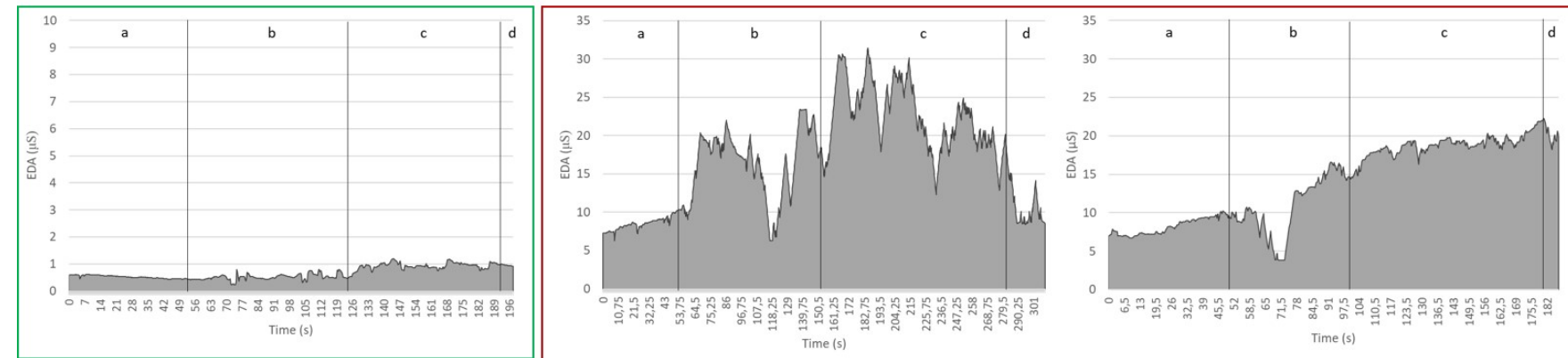

B
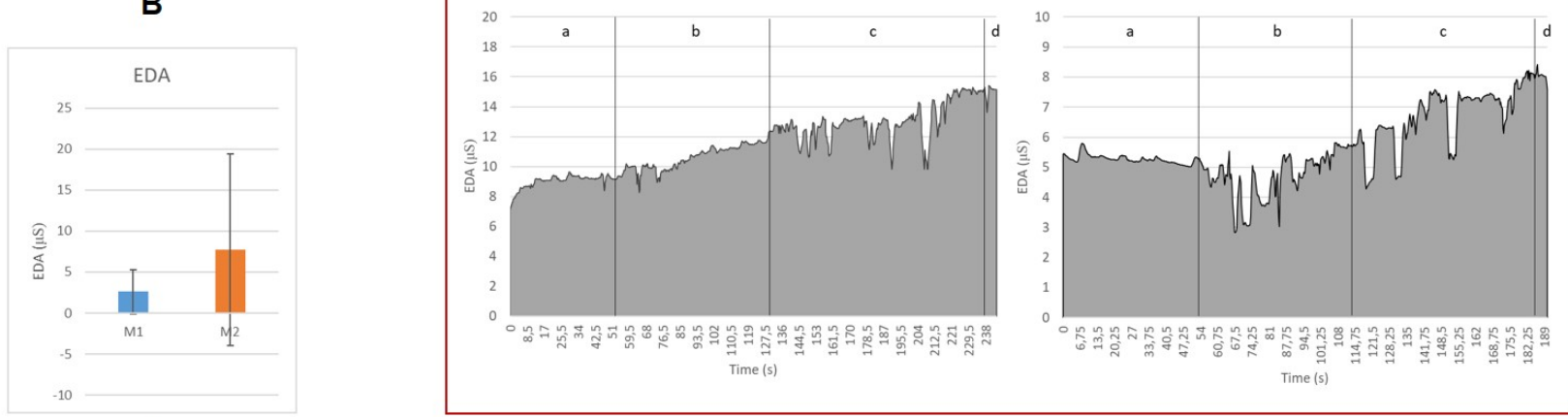

A

C

Figure 6: A: EDA values $(\mu S)$ for each modality (M1: without any vibrations; M2: with vibrations); B: EDA evolution $(\mu S)$ for one non-acrophobic participant; C: EDA evolution $(\mu S)$ for the four participants who reported being acrophobic. In $\mathrm{B}$ and $\mathrm{C}$, there was no vibration; the different phases are a: rise of the aerial device, b: ladder climbing, c: catch-a-kite task, d: end of the experiment.

\section{Discussion}

Regarding cybersickness, low scores have been observed. Therefore our simulator can be used without cybersickness interfering with the user experience. In the way the WoaH simulator has been designed, cybersickness may occur only during the motion of the virtual aerial device to the ladder, as subjects do not move physically during this time. The motion speed of the aerial device to get to the ladder is rather low both in the vertical and horizontal directions $\left(v_{\text {vert }}=0.2 \mathrm{~m} . \mathrm{s}^{-1}, v_{\text {lat }}=0.217 \mathrm{~m} . \mathrm{s}^{-1}\right.$, it takes 52 seconds to go from the ground to the ladder). The motion speed is clearly under the thresholds proposed in the literature for the occurence of cybersickness though most of past work focused on horizontal motion in virtual reality (see for example $[28,40]$ ). Also, the motion of the aerial device lasts less than one minute (the time subjects are steady on the platform and feel vibrations), which is too short to have an effect on cybersickness. When the aerial device reaches its upper position (near the ladder), the dynamic platform oscillates at a frequency of $0.3 \mathrm{~Hz}$. According to the literature, for vertical motion, oscillations that may highly provoke sickness are at frequencies about $0.2 \mathrm{~Hz}$ [2]. However, as subjects are asked to step onto the ladder as soon as the aerial device stops, there is not enough time to provoke cybersickness. Finally, when stepping onto the virtual ladder after the rise, subjects have to step physically onto a real ladder and climb it. Therefore, after stepping onto the ladder, the occurence of cybersickness due a visuo-vestibular and proprioceptive conflict that is usually observed when navigating in virtual reality should be almost non-existent.

Regarding the perceived realism, results reveal a significant effect of vibrations on the perceived realism. These results are confirmed by the feedback from the participants, who all preferred the modality including vibrations over the one without. These results are even more significant because vibrations are not set for the whole time of the experiment. Also, according to the literature, the mean score for the realism subpart of the presence questionnaire is 29.45 [33]. Here on average the scores were higher, independently from the modalities, showing that our simulator copes with the initial requirement of being realistic.

As for electrodermal activity, an increase in the EDA for the modality including vibrations was expected but no significant differences have been observed between each modality. Though on average, the EDA increased when adding vibrations, the deviation is too large to draw a strong conclusion regarding the impact of such sensory cues on anxiety. Several reasons can explain these results:

- there were only 14 participants in this first experiment, which is not enough to get a significant evolution. Experiments with many more participants should be planned;

- from Cohen's acrophobia questionnaire [13], 11 participants out of 14 got an acrophobia score under 15, which is low considering that the maximum score for this questionnaire is 120 . According to the literature, no EDA increase should therefore be found for people who do not suffer from acrophobia [1,22]. On the other hand, four participants got higher scores with the acrophobia questionnaire and for them, higher EDA variations along with tremors have been observed during the experiment;

- participants were already experienced in VR systems except one, therefore results on anxiety may have been biased. Future experiments should include future employees of the company we worked with who are expected to be totally inexperienced in VR.

Regarding the link between presence and anxiety, past research found a correlation only for acrophobic users [1]. Here, considering 
only the acrophobic participants, between both modalities, the variation of the perceived realism score seems to be positively correlated to the variation of the EDA $(r=.31)$. But again, the number of participants was too low to be significant. Nonetheless, past literature suggested that the main parameter for anxiety to develop is the perceived realism of the virtual environment [1], the perceived realism has a significant impact on presence [5], and a minimum of presence is required to develop anxiety [22], which seems to be confirmed with our simulator. We can therefore imagine that the more presence (or the more perceived realism) the better to ensure the simulator can trigger anxiety. Consequently, adding sensory cues such as vibrations is essential in a work-at-height simulator. Future studies should investigate this correlation in more detail.

\section{LIMITATIONS}

Though our simulator meets the first industrial requirements (realistic work-at-height situation, low induced cybersickness, anxiety felt by acrophobic participants), it still has some limitations, which are planned to be addressed in future work.

First, our simulator is aimed at being used indoor. Though we already provided sensory cues through the dynamic platform (motion of the aerial device and sway due to the wind), it still lacks other sensory cues that are typical from outdoor environments, that could enhance the level of immersion and anxiety. For instance, air motion could be added to simulate gusts of wind while at height. Also, the virtual scene lacks elements such as a virtual representation of the harness or of the subject's full body. These have not been added in this work for complexity reasons but they will have to be considered to enhance the level of realism. Last, most of the operations on power lines are performed with live lines. Therefore, WoaH could integrate a haptic feedback when users get close to the lines to increase stress, combined with a fear of heights.

Second, though our simulator has been designed with expert workers, in this work, we considered only non-expert subjects as test subjects. However, expert workers were asked to evaluate our simulator and provide orally feedback in terms of realism. The experts reported finding WoaH enough realistic, though they are looking forward to seeing further functionalities included as described above. Also, comparisons with reality have not been performed for safety reasons at that moment, however these are planned to make clear the effect of virtual reality, which is also a request from the expert workers. Especially, as WoaH is aimed at being used with potential future workers, experiments should be performed with applicants to work-at-height-related jobs.

\section{Conclusion}

In this paper, WoaH, a transportable virtual reality work-at-height simulator was proposed, aimed at reproducing real situations with high fidelity. An optional dynamic platform that simulates the vibrations of an aerial device's platform and its sway was included. A first user study was conducted to test whether the functionalities of our simulator met the requirements of a company dealing with work at height in terms of presence and anxiety, as WoaH aims at being deployed in such companies. Results revealed no significant relation between the modalities and cybersickness as well as the EDA, but showed a significant effect of vibrations on presence.

From this study, several guidelines can be derived for both the design and the evaluation of simulators such as the one we developed:

- according to the literature, maximizing the sense of presence among participants is essential, as a certain level of presence is a sine qua non condition for the anxiety to develop [22]. Thus, sensory modalities that provide a higher sense of presence have to be considered for such simulators; for instance, vibratory cues as presented here fit the design of a simulator involving vertical navigation, as reported also in [41].
- the evaluation of a work-at-height simulator in terms of anxiety should be performed with both acrophobic and non-acrophobic subjects. These subjects should also be inexperienced in VR.

As mentioned above, several limitations still exist on WoaH. These are planned to be addressed in future work, especially the comparison with real training without using virtual reality, mixing both experienced and inexperienced workers. Finally, future investigation should be carried out to clearly set the link between presence and anxiety in height-involving situations and clearly define which parameters encounter for both presence and anxiety in such environments.

\section{ACKNOWLEDGMENTS}

The authors wish to thank Enedis Bourgogne for their financial support and their help in designing the simulator.

\section{REFERENCES}

[1] I. Alsina-Jurnet, J. Gutiérrez-Maldonado, and M. V. Rangel-Gómez. The role of presence in the level of anxiety experienced in clinical virtual environments. Computers in Human Behavior, 27(1):504-512, 2011. doi: 10.1016/j.chb.2010.09.018

[2] A. J. Benson. Encyclopaedia of Occupational Health and Safety, chap. Motion sickness, pp. 50.12-50.40. International Labor Office, 4th ed., 1998.

[3] M. Birket-Smith, N. Hasle, and H. H. Jensen. Electrodermal activity in anxiety disorders. Acta Psychiatrica Scandinavica, 88(5):350-355, 1993. doi: 10.1111/j.1600-0447.1993.tb03471.x

[4] W. Bles, T. S. Kapteyn, T. Brandt, and F. Arnold. The mechanism of physiological height vertigo. II. posturography. Acta Oto-Laryngologica, 89(5-6):534-540, 1980. doi: 10.3109/ 00016488009127171

[5] S. Bouchard, S. Dumoulin, G. Labonte-Chartrand, G. Robillard, and P. Renaud. Perceived realism has a significant impact on the feeling of presence. Cyberpsychology \& Behavior, 9(6):660, 2006.

[6] S. Bouchard, J. St-Jacques, G. Robillard, and P. Renaud. Anxiety increases the feeling of presence in virtual reality. Presence: Teleoperators and Virtual Environments, 17(4):376-391, 2008. doi: 10. 1162/pres. 17.4.376

[7] T. Brandt, F. Arnold, W. Bles, and T. S. Kapteyn. The mechanism of physiological height vertigo. I. theoretical approach and psychophysics. Acta Oto-Laryngologica, 89(5-6):513-523, 1980. doi: 10. 3109/00016488009127169

[8] J.-R. Chardonnet, M. A. Mirzaei, and F. Merienne. Features of the postural sway signal as indicators to estimate and predict visually induced motion sickness in virtual reality. International Journal of HumanComputer Interaction, 33(10):771-785, 2017. doi: 10.1080/ 10447318.2017.1286767

[9] T. W. Cleworth, B. C. Horslen, and M. G. Carpenter. Influence of real and virtual heights on standing balance. Gait \& Posture, 36(2):172-176, 2012. doi: 10.1016/j.gaitpost.2012.02.010

[10] C. M. Coelho, J. A. Santos, C. Silva, G. Wallis, J. Tichon, and T. J. Hine. The role of self-motion in acrophobia treatment. CyberPsychology \& Behavior, 11(6):723-725, 2008. doi: 10.1089/cpb.2008.0023

[11] C. M. Coelho and G. Wallis. Deconstructing acrophobia: Physiological and psychological precursors to developing a fear of heights. Depression and Anxiety, 27(9):864-870, 2010. doi: 10.1002/da.20698

[12] C. M. Coelho, A. M. Waters, T. J. Hine, and G. Wallis. The use of virtual reality in acrophobia research and treatment. Journal of Anxiety Disorders, 23(5):563-574, 2009. doi: 10.1016/j.janxdis.2009.01.014

[13] D. C. Cohen. Comparison of self-report and overt-behavioral procedures for assessing acrophobia. Behavior Therapy, 8(1):17-23, 1977. doi: 10.1016/S0005-7894(77)80116-0

[14] H. Q. Dinh, N. Walker, L. F. Hodges, C. Song, and A. Kobayashi. Evaluating the importance of multi-sensory input on memory and the sense of presence in virtual environments. In Proceedings IEEE Virtual Reality, pp. 222-228, 1999. doi: 10.1109/VR.1999.756955

[15] P. M. Emmelkamp, M. Bruynzeel, L. Drost, and C. A. van der Mast. Virtual reality treatment in acrophobia: a comparison with exposure 
in vivo. CyberPsychology \& Behavior, 4(3):335-339, 2001. doi: 10 . 1089/109493101300210222

[16] H. Hsiao, P. Simeonov, B. Dotson, D. Ammons, T.-Y. Kau, and S. Chiou. Human responses to augmented virtual scaffolding models. Ergonomics, 48(10):1223-1242, 2005. doi: 10.1080/ 00140130500197112

[17] R. Hüweler, F. I. Kandil, G. W. Alpers, and A. L. Gerlach. The impact of visual flow stimulation on anxiety, dizziness, and body sway in individuals with and without fear of heights. Behaviour Research and Therapy, 47(4):345-352, 2009. doi: 10.1016/j.brat.2009.01.011

[18] D. P. Jang, J. H. Ku, Y. H. Choi, B. K. Wiederhold, S. W. Nam, I. Y. Kim, and S. I. Kim. The development of virtual reality therapy (VRT) system for the treatment of acrophobia and therapeutic case. IEEE Transactions on Information Technology in Biomedicine, 6(3):213-217, 2002. doi: 10.1109/TITB.2002.802374

[19] M. C. Juan and D. Pérez. Comparison of the levels of presence and anxiety in an acrophobic environment viewed via HMD or CAVE. Presence: Teleoperators and Virtual Environments, 18(3):232-248, 2009. doi: $10.1162 /$ pres. 18.3 .232

[20] R. S. Kennedy, N. E. Lane, S. Kevin, and M. G. Lilienthal. Simulator Sickness Questionnaire: An enhanced method for quantifying simulator sickness. The International Journal of Aviation Psychology, 3(3):203220, 1993. doi: 10.1207/s15327108ijap0303_3

[21] Y. Y. Kim, H. J. Kim, E. N. Kim, H. D. Ko, and H. T. Kim. Characteristic changes in the physiological components of cybersickness. Psychophysiology, 42(5):616-625, 2005. doi: 10.1111/j.1469-8986. 2005.00349.x

[22] M. Krijn, P. M. G. Emmelkamp, R. Biemond, C. de Wilde de Ligny, M. J. Schuemie, and C. A. P. G. van der Mast. Treatment of acrophobia in virtual reality: The role of immersion and presence. $B e-$ haviour Research and Therapy, 42(2):229-239, 2004. doi: 10.1089/ 109493101300210222

[23] J. H. Kwon, J. Powell, and A. Chalmers. How level of realism influences anxiety in virtual reality environments for a job interview. International Journal of Human-Computer Studies, 71(10):978 - 987. 2013. doi: 10.1016/j.ijhcs.2013.07.003

[24] C. Lai, R. P. McMahan, and J. Hall. March-and-reach: A realistic ladder climbing technique. In 2015 IEEE Symposium on 3D User Interfaces (3DUI), pp. 15-18, 2015. doi: 10.1109/3DUI.2015.7131719

[25] C. McCarthy, N. Pradhan, C. Redpath, and A. Adler. Validation of the empatica E4 wristband. In 2016 IEEE EMBS International Student Conference (ISC), pp. 1-4, 2016. doi: 10.1109/EMBSISC.2016. 7508621

[26] M. Meehan, B. Insko, M. Whitton, and F. P. Brooks, Jr. Physiological measures of presence in stressful virtual environments. $A C M$ Transactions on Graphics, 21(3):645-652, 2002. doi: 10.1145/566654. 566630

[27] M. Meehan, S. Razzaque, M. C. Whitton, and F. P. Brooks. Effect of latency on presence in stressful virtual environments. In IEEE Virtual Reality, pp. 141-148, 2003. doi: 10.1109/VR.2003.1191132

[28] M. A. Mirzaei. Influence of interaction techniques on VIMS in virtual environments: estimation and prediction. $\mathrm{PhD}$ thesis, Ecole Nationale Supérieure d'Arts et Métiers, 2014.

[29] J. Plouzeau, J.-R. Chardonnet, and F. Merienne. Dynamic Platform for Virtual Reality Applications. In EuroVR, pp. 2-5, 2017.

[30] J. Plouzeau, J. L. Dorado, D. Paillot, and F. Merienne. Effect of footstep vibrations and proprioceptive vibrations used with an innovative navigation method. In IEEE Symposium on 3D User Interfaces (3DUI), pp. 241-242, 2017. doi: 10.1109/3DUI.2017.7893361

[31] M. S. Redfern, J. M. Furman, and R. G. Jacob. Visually induced postural sway in anxiety disorders. Journal of Anxiety Disorders, 21(5):704-716, 2007. doi: 10.1016/j.janxdis.2006.09.002

[32] H. T. Regenbrecht, T. W. Schubert, and F. Friedmann. Measuring the sense of presence and its relation to fear of heights in virtual environments. International Journal of Human-Computer Interaction, 10(3):233-249, 1998. doi: 10.1207/s15327590ijhc1003_2

[33] G. Robillard, S. Bouchard, P. Renaud, and L. G. Cournoyer. Validation canadienne-française de deux mesures importantes en réalité virtuelle : l'Immersive Tendencies Questionnaire et le Presence Questionnaire. In 25e congrès de la Société Québécoise pour la Recherche en Psychologie (SQRP), 2002.

[34] B. Rothbaum, L. Hodges, R. Kooper, D. Opdyke, J. Williford, and M. M. North. Effectiveness of computer-generated (virtual reality) graded exposure in the treatment of acrophobia. American Journal of Psychiatry, 152(4):626-628, 1995. doi: 10.1176/ajp.152.4.626

[35] P. I. Simeonov, H. Hsiao, B. W. Dotson, and D. E. Ammons. Height effects in real and virtual environments. Human Factors, 47(2):430438, 2005. doi: 10.1518/0018720054679506

[36] R. Skarbez, F. P. Brooks, Jr., and M. C. Whitton. A survey of presence and related concepts. ACM Computing Surveys, 50(6):96:1-96:39, 2017. doi: $10.1145 / 3134301$

[37] M. Slater. Place illusion and plausibility can lead to realistic behaviour in immersive virtual environments. Philosophical Transactions of the Royal Society of London B: Biological Sciences, 364(1535):3549-3557, 2009. doi: $10.1098 /$ rstb. 2009.0138

[38] M. Slater, M. Usoh, and A. Steed. Steps and ladders in virtual reality. In Proceedings of the Conference on Virtual Reality Software and Technology (VRST '94), pp. 45-54, 1994.

[39] M. Slater and S. Wilbur. A Framework for Immersive Virtual Environments (FIVE): Speculations on the role of presence in virtual environments. Presence: Teleoperators and Virtual Environments, 6(6):603-616, 1997. doi: 10.1162/pres.1997.6.6.603

[40] R. H. Y. So, W. T. Lo, and A. T. K. Ho. Effects of navigation speed on motion sickness caused by an immersive virtual environment. Human Factors, 43(3):452-461, 2001. doi: 10.1518/001872001775898223

[41] K. Vasylevska and H. Kaufmann. Influence of vertical navigation metaphors on presence. In Challenging Presence - Proceedings of 15th International Conference on Presence (ISPR 2014), pp. 205-212, 2014.

[42] B. G. Witmer and M. J. Singer. Measuring presence in virtual environments: A presence questionnaire. Presence: Teleoperators and Virtual Environments, 7(3):225-240, 1998. doi: 10.1162/105474698565686 